\title{
PROSTITUTION COLONIALE ET NATIONALISME: COLLABORATION CORPORELLE DANS CIEL DE PORPHYRE DE AICHA LEMSINE Guettafi SIHEM Soltani WASSILA
}

"Comprendre que la sexualité est politique implique de reconnaître que la domination est implantée au cœur même du fantasme..."

(MOLINIER, 2009: 254)

RÉSUMÉ : Les prostituées ont été utiles dans la période coloniale de l'Algérie, car, d'une part, la sexualité représentait pour le colonisateur français l'une des formes les plus pernicieuses de la domination et un élément fondamental d'assujettissement : ces femmes algériennes indigènes devenaient un réel sujet de l'imaginaire érotique colonial. Et d'autre part, ces femmes représentaient pour les nationalistes combattants algériens une véritable source de renseignements au profit du FLN (c'est le partie politique : Front de libération nationale qui a déclenché la guerre d'Algérie de 1954) car elles étaient en contact régulier avec des policiers ou des militaires français. Ces femmes n'étaient pas des objets passifs mais de vérita-

\footnotetext{
${ }^{1}$ Maître assistant en littérature. Université de Biskra (Algérie) - mail : guetfsihem@yahoo.fr 2 Doctorante en didactique. Université de Biskra (Algérie) - mail : soltani.silla@yahoo.fr
} 
bles actrices, pensantes et agissantes. De Dalila, la prostituée au service du colonisateur à Houria prostituée, ange gardien et libératrice des pulsions accumulées par les moudjahiddines. Ciel de Porphyre d'Aicha Lemsine s'ouvre et se termine par une lecture des actions de prostituées durant la guerre de libération algérienne. Notre réflexion tournera autour du rôle de la femme algérienne « indigène » qui a participé à la lutte armée en vendant son corps et en subissant des violences sexuelles pour servir la bonne cause. De démontrer comment Aicha Lemsine à travers son roman, "Ciel de porphyre », traite-t-elle ces actions de prostituées patriotes, c'est-à-dire, au service de leur pays : l'Algérie libre et indépendante ? Comment dans le roman « Ciel de porphyre » l'équation Nationalisme=prostitution est-elle perçue ? Comment l'auteur(e) établit- elle cette collaboration corporelle et charnelle ? Et enfin Comment mesure -t-elle l'ampleur de cette collaboration entre filles "indigènes/ algérienne » et « européens/français colonisateurs »?

MOTS-CLÉS : Prostitution coloniale, Fiction, Femmes, Littérature, Nationalisme.

\section{PROSTITUIÇÃO COLONIAL E NACIONALISMO: COLABORAÇÃO CORPORAL EM CIEL DE PORPHYRE DE AICHA LEMSINE}

RESUMO: As prostitutas eram úteis no período colonial, pois, por um lado, a sexualidade representava para o colonizador francês uma das formas mais perniciosas da dominação e um elemento fundamental de submissão: estas mulheres argelinas indígenas se tornavam um verdadeiro tema do imaginário erótico colonial. E, por outro lado, estas mulheres representavam para os nacionalistas combatentes argelinos uma verdadeira força de informações para profeito da FLN (o partido político: Fronte de Liberação Nacional que desencadeou a guerra da Argélia de 1954), pois elas ficavam em contato regular com policiais ou militares em exercício. De Dalila, a prostituta a serviço do colonizador, a Houria, prostituta, anjo guardião e libertadora das pulsões acumuladas pelos moudjahiddinos, Ciel de Porphyre de Aicha Lemsine se abre e se encerra por uma leitura das ações de prostitutas durante a guerra de liberação argelina. Nossa reflexão se voltará para o papel da mulher argelina indígena que participou da luta armada vendendo seu corpo e enfrentando violências sexuais para servir à boa causa, demonstrando como Aicha Lemsine, através de seu romance Ciel de Porphyre, trata as ações de prostitutas patriotas, isto é, a serviço de seu país: a Argélia livre e independente? Como no romance Ciel de Porphyre a equação Nacionalismo - prostituta é percebida? Como a autora estabelece esta colaboração corporal e carnal? E, finalmente, como medir o alcance dessa colaboração entre jovens indígenas/argelinas com europeus / colonizadores franceses?

PALAVRAS-CHAVE: Prostituição colonial, Ficção, Mulheres, Literatura, nacionalismo. 
Pour M. Foucault, le corps est un enjeu politique. Il est aussi un enjeu de l'ordre du colonial. Dans quelles mesures, alors, le corps est-il un enjeu politique ? Comment la problématique coloniale peut-être lue à travers le corps ? De manière plus précise : la colonisation a-t-elle un corps ?

Le corps est une idée neuve dans l'histoire, c'est un lieu d'expression. Voilé ou dévoilé, souffrant ou violé, torturé ou glorieux, le corps a été de tout temps objet de représentations. Représentation artistique surtout et par là un moyen d'influence sur les esprits. Les représentations occidentales font du corps « oriental / indigène » un corps exotique par excellence. L'ordre colonial passe alors par un ordre des corps : le corps est politisé « la politique sexuelle est toujours une politique du corps. D'une part, c'est le corps qui manifeste, proteste, qui se bat. Et d'autre part, c'est le corps qui est l'enjeu de la lutte »3.

Dans le processus de la colonisation, deux corps s'affrontent: le corps du colonisateur et le corps du colonisé. Dans le contexte colonial, le corps du colonisé « indigène », en plus d'être violenté quotidiennement à travers les exécutions sommaires, les violences psychologiques, les viols, il est dévirilisé et bestialisé. Le corps « indigène » est un corps souffrant, affamé, dégénérescent ; il est sexualisé et racialisé. Celui de la femme «indigène » est sexualisé et érotisé à son tour. Le colonisateur en s'attaquant et violentant le corps "indigène » de cette manière, nous a permis de s'interroger si la colonisation avait un sexe. Le colonisateur serait à l'image de l'homme viril qui valorise la domination et la France serait l'image de la supériorité sexuelle du colon qui domine l'Algérie, femme faible, dominée et sexuellement inférieure mais désirée. En effet, la France "virile » liait sexualité et violence, domination masculine et domination coloniale.

Cette notion du corps en lutte nous oblige à revenir un peu en arrière pour découvrir les éléments déclencheurs qui ont dévoilé que le corps féminin est un enjeu politique, lieu où se jouent des batailles politiques et idéologiques cruciales. « De l'orientalisme à l'iconographie coloniale, les femmes mauresques/ orientales étaient présentées, au début de la colonisation, voilées à travers les tableaux de peintures ou la photographie coloniale vers 1860 puis vers 1910, elles sont montrées nues »4. On passe progressivement de l'idée de voilement à l'idée du dévoilement/du nu. Les représentations symboliques sont omniprésentes, la question de la sexualité féminine l'est aussi.

Ce passage mènera à la naissance d'une vraie « économie du sexe » et à travers elle à la prostitution coloniale 5 . Un processus de dégradation du corps de la femme orientale/indigène transparaît dans ce projet, l'objectif est de faire des femmes et de leurs corps des objets au servir de l'ordre colonial. La femme devient un véri-

\footnotetext{
${ }^{3}$ Conférence de DIDIER ERIBON, professeur à la faculté de philosophie, sciences humaines et sociales de l'université d'Amiens. Le corps politique. Quelques réflexions à propos du $40^{\text {ème }}$ anniversaire des émeutes de Stonewall.

${ }^{4}$ TARAUD, Christelle. La prostitution coloniale. Algérie, Tunisie, Maroc (1830-1962). Paris, Payot, 2003

${ }^{5}$ lbid.
} 
table enjeu dans la conquête et dans la révolution plutard : c'est le territoire de combat par excellence. La femme et son corps sont symboliquement l'épicentre à partir duquel naît l'action révolutionnaire : ces deux entités seraient le moteur de toute révolution. Elle a été à la fois victime et actrice de grands mouvements révolutionnaires, elle occupe une place importante durant la période coloniale.

Notre réflexion visera à démontrer quelle place occupe les femmes au milieu des secousses révolutionnaires qui ont bouleversé l'univers maghrébin et algérien, en particulier. Comment la femme algérienne a-t-elle perçue, vécue, contribuée à la révolution ? Comment a-t-elle participé à la lutte armée en utilisant son corps comme arme de combat? Quelles violences sexuelles a-t-elle subit pour servir la bonne cause ? Comment la fiction lemsenienne traite-t-elle les actions de prostituées ? Et comment définit-elle l'équation Nationalisme $=$ Prostitution en traitant cette collaboration corporelle et charnelle?

Pour répondre à ce flot de questionnements, nous commencerons par le début. Des orientalistes qui ont fantasmé sur le corps « oriental/indigène », du mouvement exotique qui a permis à ce fantasme de devenir réalité en s'appropriant et possédant, enfin, le corps lointain, interdit et caché tant désiré. Cette possession corporelle et sexuelle s'est transformée en enjeux politiques. Le corps des femmes est devenu un véritable champ de bataille et une politique sexuelle va voir le jour dès les premiers moments de la conquête. En effet, la colonisation a construit une image particulière des femmes du Maghreb, cette construction s'est effectuée par étapes, à mesure que l'expansion coloniale gagnait de plus en plus la terre algérienne.

La peinture et la littérature des orientalistes ont permis de découvrir une image de la conquête: une image de l'altérité féminine, une altérité romantique et exotique où les femmes sont omniprésentes parce qu'elles évoquent comme l'écrit Fromentin " un royaume inviolable du soleil et de la lumière " 6 . Ces artistes vont mettre en scène un univers où les femmes sont exhibées dans des décors qui les présentaient comme des divinités envoûtantes. Fromentin ou Delacroix ont tenté de les montrer comme un mirage de vraisemblance difficile d'approcher. Les femmes « orientales » obsédaient les orientalistes, elles sont l'un des mystères insondables des Médinas traditionnelles auxquelles elles sont symboliquement associées. Les orientalistes expriment, dès lors, dans la figure de la femme voilée qui apparait comme une parfaite métaphore de l'inconnue à la face cachée, la tentatrice et désirable qui joue à la dissimulation.

Donc, la tentation de l'orient, n'est pas seulement l'attrait des contrées lointaines à explorer et découvrir, c'est aussi une véritable quête des plaisirs charnels. Les colonies apparaissent comme « l'éden sexuel des occidentaux » avec leurs harems « réprimant jour après jour leurs désirs, les européens se défoulaient dans les colonies imaginaires »7. Ce sont la peinture et la littérature qui vont propager et entretenir ce mirage. On citera, à titre d'exemple, Fromentin se ren-

\footnotetext{
${ }^{6}$ FROMENTIN, Eugène. Une année dans le Sahel. Paris : Ed Plon, 1848.

7 TARAUD, Christelle. Op. cit.
} 
dant chez Haoua, courtisane blidéenne (ville de Blida en Algérie) en se demandant si son chez elle ressemble à celui des femmes d'Alger dans leurs appartements de Delacroix. Ou encore de l'extraordinaire description que fait Emile Masqueray de la danseuse Khamissa, (dans ses souvenirs de vision d'Afrique). Cette idée largement véhiculée aboutit à une équation très simple, qui consiste à faire de chaque femme " orientale/ indigène » une femme aux mœurs légères donc une prostituée potentielle affirmait en 1912 Christian Houel.

Cette idée ne représente pas la réalité, mais une vision fantasmatique des femmes « orientales/indigènes » qui, entre la vision et l'histoire artistique d'une féminité orientale et l'histoire politique de la prise de possession de cette féminité, participe largement de la domination coloniale. La prostituée «indigène » est l'archétype de la femme fantasmée. Pour comprendre la vision coloniale et en quoi l'équation "femmes indigènes = mœurs faciles= prostituées ", il faut comprendre que la sexualité est l'une des formes des plus pernicieuses de la domination : la colonisation sexuelle des femmes réelles ou symboliques est un facteur essentiel de contrôle de l'assujettissement.

La production littéraire et iconographique coloniale traduisent, justement, cet état de fait en proposant dans la plupart des cas des relations sexuelles intercommunautaires. Ce sont des scènes comme : des hommes blancs qui ont des aventures avec des femmes «indigènes »; cette production met, donc, en scène un rapport de force qui s'exerce, plus ou moins violemment selon les cas, au détriment de la société colonisée, tout en évoquant aussi une forme ambiguë de désir de l'autre faite d'attraction et de répulsion. L'imaginaire colonial s'est, dès lors, nourri de ces nombreuses représentations de femmes « indigènes » dans des postures fantasmatiques importées par la colonisation. Cette vision des postures fantasmatiques, n'est à l'origine qu'une fiction inventée, imaginée par les artistes orientalistes du 19 ès et du début du $20^{\mathrm{èS}} \mathrm{S}$ au sein des Harems et hammams.

L'idée de départ est comment faire mieux cultiver l'idée que l'on se fait de l'orient, comment mieux faire rêver l'Europe, la France « virile » qu'en exposant des femmes orientales réputées inaccessibles dans le lieu privilégié de leurs appartements. Comment décrire ces belles recluses dans leurs lieux clos, mais ouverts à tous les délices permettant de produire des images sexuellement attractives.

Ce n'est pas la vie réelle des femmes cloîtrées dans leur enfermement et solitude qui intéresse ces artistes, mais la vie sublimée, désincarnée et érotisée à l'extrême. La peinture orientaliste est la traductrice romanesque de cette fascination. L'orientalisme impose, donc, l'image d'une féminité oisive, passive et surtout offerte ${ }^{8}$.

${ }^{8}$ Ibid. 
La représentation à l'intérieur des scènes de harems et de hammams, de femmes orientales sexuellement soumises et suggestives obéit (selon des études) à des canons généralement européens sur le plan de: l'architecture, mobiliers, vêtements, éléments formant le décor des peintures orientalistes. Ajoutons à cela, la caractérisation physique des femmes peintes: clarté des cheveux, blancheur immaculée des peaux et minceur des corps ce qui n'est pas en adéquation avec les normes de la beauté orientale (cheveux noirs, peaux mates et corpulence) ${ }^{9}$. La question que se pose (Christelle Taraud, 2003) est : pourquoi la plupart des artistes orientalistes peignent-ils avec une telle constance des européennes travesties en orientales? (Notons que : sauf Etienne Dinet et Jacques Majorelle donnent une image plus réaliste des femmes maghrébines).

Il est probable que cette représentation factice de la différence transfigure d'une part une profonde frustration vis-à-vis des femmes européennes. D'autre part, explique C. Taraud l'exposition des occidentales à l'intérieur des Harems conforte une certaine idée de supériorité raciale fortement insérée dans le projet colonial. Dévoilant par là, une idée très prégnante dans l'imaginaire érotique occidental : celle de la "virilité triomphante du maître ». Ces orientalistes, en entreprenant toute une archéologie des fantasmes orientaux, à travers des visions hautement fantasmatiques, font apparaitre un désir exclusif de soi qui ne peut être exprimé sans la médiation de l'autre.

En plus, de la peinture, la photographie coloniale fait des femmes « indigènes » un véritable sujet de l'imaginaire érotique colonial. Malek Alloula, dans son brûlant réquisitoire postcolonial contre la carte postale en Algérie, parle de " revanche imaginaire contre des femmes inaccessibles ${ }^{10}$. Selon lui, les femmes présentées dans les photographies ou cartes postales ne sont que des métaphores de l'impuissance coloniale. Sachant par le colonisateur que ces images menacent l'identité virile des colonisés. Il faut voir en ces images une forme particulière de domination qui consiste pour le colon à usurper symboliquement le pouvoir de contrôle des femmes des autres « les indigènes ». «... Ses salariées de la pose aux tristes visages sont les avatars dégradés d'un colonialisme proxénète et prédateur, mais elles sont aussi le produit d'une société à droits limités où la prostitution des femmes est une condition de survie $»^{11}$.

La question de la violence sexuelle coloniale est revenue ces dernières années sur le devant de la scène de manière inattendue, mais avec force. C'est grâce au témoignage de Djamila Boupacha et au calvaire qu'elle avait subi en 1960 lors de sa torture, qu'un passé colonial mal assumé et refoulé remontait à la surface, dévoilant le calvaire et les souffrances vécus par les femmes durant la colonisation : femmes violées ou femmes soumises, elles mettent à nu le passé d'une France « vi-

\footnotetext{
${ }^{9}$ MONTAGU, Lady Mary, L'islam au péril des femmes nues. Une Anglaise en Turquie au $18^{\mathrm{è}} \mathrm{S}$, 2001, p.103.

${ }_{10}$ ALOULA, Malek, Le Harem colonial. Images d'un sous érotisme. Ed Séguier: Paris, $1981.1^{\text {ère }}$ Ed. p. 78.

${ }^{11}$ lbid.
} 
rile » qui liait sexualité et violence, domination masculine et domination colonia$\mathrm{le}^{12}$. Cette violence sexuelle est devenue structurée grâce à une politique d'exception (l'indigénat) activée pour créer un domaine prostitutionnel prospère. En effet, l'organisation de la prostitution en Afrique du Nord est apparue avec l'implantation d'un système répressif, entraînant avec elle une véritable " économie du sexe » d'une ampleur et d'une nature insolite et explosive de « racialisme » et de « capitalisme».

Ceci dévoile une véritable colonisation sexuelle des femmes «indigènes " créant un réel réseau prostitutionnel maghrébin. En effet, c'est à l'aube de la colonisation qu'un contexte prostitutionnel spécifiquement Nord-africain est né. Cet univers prostitutionnel maghrébin antérieur à la colonisation n'existait pas. C'est une construction exclusivement coloniale, affirmaient les historiens de la décolonisation. Pour d'autres, elle n'est pas seulement dérangeante mais fausse, car des archives repérées ont montré qu'une sexualité vénale intercommunautaire à destination d'une population étrangère et conquérante, antérieur à la colonisation française, existait déjà durant la période ottomane. Avant la conquête française en Algérie, la ville d'Alger se présentait comme une juxtaposition de quartiers organisés selon un véritable souci de l'ordre public.

La ville est, par ailleurs, divisée en deux parties distinctes : «la montagne » où vivait la majorité de l'élite citadine (les mauresques) et en bas « la plaine » qui s'étend jusqu'à la mer où la population est cosmopolite et la vie plus animée, c'est dans cette basse ville que se trouve le quartier « Franc », peuplé à cette époque d'une centaine de chrétiens pour la plupart fonctionnaires consulaires. L'existence des européens est considérée par les citadins de la «montagne » comme signe de malédiction, car ils s'adonnaient à des pratiques non désirées tels l'alcoolisme et la prostitution.

Avec la conquête, l'arrivée massive des militaires à Alger accentue le mouvement, surtout avec la destruction des medersas et des mosquées (en 1836, six mosquées avaient été détruites) pour loger les soldats car l'effectif de l'armée de l'Afrique est passé, pour lutter contre L'émir Abdelkader, de 37.00o hommes en 1830 à 100.000 hommes en 1847. C'est dans ce flot continu de migrants venant de toute l'Europe que se glissent les premières prostituées : italiennes, maltaises, françaises et espagnoles. C'est à partir de cette nouvelle arrivée que s'organisent les premiers trafics de prostitution. Cette prostitution nouvelle, qui se répand rapidement, répond à une demande spécifique des européens. C'est dans les lieux bruissant et mélangés de la Médina que se pratiquent les jeux de hasard, l'alcoolisme et la prostitution avec des filles publiques. La réglementation coloniale met, alors, en adéquation « sans distinction de nations », la prostitution locale et traditionnelle : « indigène » avec la prostitution « migrante / européenne ».

Il faut préciser que le fait prostitutionnel maghrébin à l'époque est né de la combinaison d'une insécurité familiale récurrente et d'une précarité économique

\footnotetext{
${ }^{12}$ BEAUGE, Florence, « Le tabou du viol des femmes pendant la guerre d'Algérie Commence à être levé ». Le Monde, 11 octobre 2001.
} 
qui est l'un des principaux vecteurs du passage à la prostitution. Il existe différents types de rencontre, dans ce contexte colonial. Dans un premier temps, celle des militaires français avec des prostituées européennes. Puis celle des militaires français avec des prostituées « indigènes ». Ces rencontres ne sont pas coupées de leur contexte de production : celui de la colonisation. Ces prostituées, surtout les « indigènes » se trouvent pris dans un entre-deux : entre soumission et émancipation, entre tradition et modernité, entre ordre et désordre.

Une question pourtant s'impose à nous : comment le volet prostitutionnel s'est structuré en Algérie après la conquête ? Un petit historique s’impose. En 1831, après la conquête, un document est crée portant sur la création d'un établissement pour la visite sanitaire des filles publiques. L'organisation d'un service des mœurs est complétée en 1835 . Ce volet est entré en vigueur dans ce projet sanitaire dès les années 1871 à la fin de la révolte de Mokrani. En 1898, un observateur note:

La prostitution en Algérie est une plaie qui, depuis une quinzaine d'années, a pris des proportions telle qu'elle réserve dans avenir prochain un état de choses qu'il deviendra impossible d'enrayer. Quoi que colonie, l'Algérie est trop près de la France pour que les effets de la prostitution qui s'y exerce trop librement, ne produisent les résultats les plus désastreux...

Ce n'est qu'en 1953 que la commission municipale achève d'imprimer un code légal à la prostitution publique restaurant un lieu d'exercice privilégié. Cette réglementation va permettre la création des premiers quartiers réservés exclusivement à des prostituées « indigènes ». " Le paroxysme de cette politique est atteint au Maroc avec la création, au début des années 1920, de véritables citées prostitutionnelles telle Bousbir à Casablanca »13. Selon Norbert Elias, qui en fait un portrait très précis, « la civilisation des mœurs » aboutit en Afrique du Nord à la construction d'une prostitution utilitaire, moderne et visible qui canalise et rend productive toute énergie, y compris l'énergie sexuelle ${ }^{14}$.

Quels sont les critères d'implantation des maisons closes ? En Afrique du Nord, les critères d'implantation sont rapidement précisés par la législation, car l'administration coloniale redoute le développement anarchique des maisons de tolérance. Ce sont des consignes strictes qui sont mises en place sur le modèle parisien du règlement Gigot en $1878^{15}$. Ce recueil présente des textes appliqués, à quelques variantes près, dans l'ensemble de l'Afrique du Nord. Il précise qu'une maison de tolérance ne peut-être ouverte, aménagée, exploitée, transformée, transférée qu'avec l'accord du préfet, après avis et enquête de l'inspecteur dépar-

${ }^{13}$ TARAUD, Christelle, Op. cit.

${ }^{14}$ NORBERT, Elias, La Société de cours. Paris : Champs essais, 1985. p. 47.

${ }^{15}$ CORBIN, Alain. Les Filles de noces. Misère sexuelle et prostitution au $19^{\grave{e}} \mathrm{~s}$ et au $20^{\mathrm{è}} \mathrm{s}$. Paris : Flammarion, 1978. p. 87. 
temental d'hygiène. Aucune maison ne peut-être installée dans le voisinage d'un établissement public, ou privé, d'une école, d'un hospice, d'un édifice de culture, d'une caserne ou d'une prison (c'est l'administration préfectorale qui se chargera de fixer les distances à imposer. Cette distance était fixée à $300 m$ dans la réglementation française).

La demande d'ouverture et d'exploitation d'une maison de tolérance doit être faite sur un papier timbré et adressé au préfet accompagné d'un plan de l'immeuble et du quartier. Elle indique aussi le nombre de pensionnaires que la maison doit héberger. De plus, l'autorisation d'ouverture ne peut-être accordée qu'à une femme âgée de 25 ans passés (selon l'arrêté du 30 avril 1942). Cette réglementation vise surtout à réguler l'implantation de la prostitution réglementée européenne (jusqu'aux années 1900) ainsi que des maisons de tolérance européennes et indigènes.

A Alger, en 1859, les quatorze maisons publiques répertoriées par le docteur Bertherand étaient toutes européennes. En 1930, un siècle après la création de la première maison close, l'Algérie compte seulement 68 établissements de plaisir européens (TARAUD, 2003).

\begin{tabular}{|c|c|c|c|}
\hline Années & lieux & Maisons « européenne » & Maisons « indigènes » \\
\hline 1859 & Alger & 14 avec 189 prostituées & 1 \\
\hline 1905 & Alger & 15 & 17 \\
\hline 1930 & Alger & 22 pour les deux & \\
\hline 1930 & $\begin{array}{l}\text { Constantine } \\
\text { Oran } \\
\text { Biskra } \\
\text { Laghouat }\end{array}$ & $\begin{array}{l}28 \text { pour les deux } \\
18 \text { pour les deux } \\
2 \text { européennes } \\
2 \text { européennes }\end{array}$ & \\
\hline 1935 & $\begin{array}{l}\text { Alger } \\
\text { Quartier rouge } \\
\text { de la Casbah }\end{array}$ & o5 dont le légendaire sphinx & 35 \\
\hline
\end{tabular}

La prostitution réglementée ne s'implante que sur une large bande comprise entre Alger, Tunis et Tanger. Au-delà de cette bande, les prostituées "indigènes » sont quasiment partout en situation de monopole sauf à Laghouat et Biskra où ne sont implantées que des maisons de tolérance européennes. C'est, en effet, dans les trois départements français d'Algérie où la présence coloniale est la plus abondante et la plus ancienne qu'on y trouve le plus grand nombre de maisons de tolérance de première et deuxième catégorie implantées surtout dans les grands boulevards avec une clientèle de prestige composée d'officiers, de grands commis, de chefs d'entreprises et de gros colons.

Mais c'est dans les quartiers chauds que les maisons de tolérance sont les plus enracinées, pour une clientèle pauvre, de simples militaires. Les tenancières européennes tentent de copier les maisons de tolérance parisienne, même les 
noms de ces établissements sont français comme «le chat noir » de Bône, le Sphinx de la Casbah (sont attachés à la métropole). Germaine Aziz décrit dans les années 1950 l'établissement le plus réputé d'Alger "le Sphinx » et sa tenancière madame Nana, qui est «très fière de sa maison, l'en fait un bordel "select", elle affirme qu'il est connu jusqu'à Paris ${ }^{16}$. Nous trouvons ces nominations dans notre fiction Ciel de porphyre.

«C'est en 1956, la guerre secouait l'Algérie, à Baladia "ville portuaire" » (Ciel de Porphyre ${ }^{17}$, p. 78) où Ali, notre Héros, eu sa première initiation sexuelle. Les jours de semaine, il étudiait mais les jours de sortie, il se rendait chez un ami de son père pour y déposer ses affaires et prendre ses repas. Il passait le plus grand temps avec ses amis qui le taquinaient souvent sur sa chasteté. Un jour en gagnant « un match mémorable, Slimane, son ami voulait lui faire un cadeau » (CP, p. 135). «Lui offrir une femme, dans une grande maison" (CP, p. 135). "C'est ainsi que les quatre amis (Slimane, Mahmoud, Fernand et Ali) se rendent "au cheval blanc", lieu d'initiation aux plaisirs de la vie, connu pour être une maison spécialisée dans les femmes Arabes» (CP, p. 136). "Les femmes sont vêtues de longues gandouras à volant sur le bas et autour du buste, avec leur foulard noué coquettement autour de la tête et leur yeux soulignés de Khôl, elles ressemblent à n'importe quelle mère, ou fille de famille » (CP, p. 136). Ali eu soudain envie de fuir car «ces femmes avec leurs gandouras...me rappelèrent subitement ma mère... » (CP, p. 136.).

Ali se dirige vers la sortie, suivi de ses amis qui l'emmenèrent de force dans un autre établissement « La souris noir » (qui se rapproche par le nom au fameux « Chat noir » de Bône cité par Germaine Aziz, ancienne prostituée juive durant la période coloniale, dans l'histoire de la prostitution coloniale de Christelle Taraud). Maison close abritant des prostituées européennes. Le décor de « la souris noir » rappelle étrangement celui des salons parisiens «ici, c'est différent! Un grand salon avec au fond un bar impressionnant, les murs décorés avec des tableaux représentant des femmes dévêtues... » (CP, p. 138). «La patronne, une grosse femme appelée madame Nini » (CP, p. 138) qui renvoi à madame Nana, tenancière du fameux Sphinx d'Alger vers les années 1950.

Ce qui fait parisien et français des bordels de premières catégories implantées dans les grandes villes, témoigne ainsi d'une démarcation jugée évidente même dans le monde de la prostitution. Entre « le civilisé » et le "sauvage », entre « le colon » et "l'indigène », les maisons de tolérance sont, en effet, théoriquement tenues par une jurisprudence non officielle, de respecter une certaine ségrégation raciale et religieuse dans le choix de leur clientèle. Par exemple à Constantine, en 1930, la maison close le «Belvédère » se démarque par le fait qu'elle est rigoureusement interdite aux «indigènes ». Selon Roger Salardenne, cette interdiction «permet aux français de ne pas utiliser les laissées pour compte des

${ }^{16}$ GERMAINE, Aziz. Les Chambres closes. Paris : Ed Stock, 1980.

${ }^{17}$ Dorénavant: CP. 
Arabes » car « il est assez humiliant et désagréable pour un blanc de songer qu'il a été précédé dans les bras d'une femme, même prostituée, par un Sidi à la santé douteuse et à l'hygiène plus ou moins relative. ${ }^{18}$.

On interdisait aux femmes musulmanes de fréquenter des infidèles, les prostituées «indigènes » sont censées rejeter les relations intimes avec les chrétiens. A Tunis, par exemple, dans les années 1930, les filles soumises « indigènes » ne "marchaient qu'avec les Arabes, ni les européens, ni les Israélites, n'étaient admis à profiter de leurs charmes, les règles étaient rigoureuses. Il ne fallait pas mélanger les races»(selon Salardenne). Les maisons de tolérance européennes sont concurrencées par certains établissements « indigènes » de bonne tenue, ils possédaient une clientèle exclusivement européenne. La compétition se fait de plus en plus féroce dans ces lieux populaires. Cet écart entre les deux maisons s'explique sans doute par la capacité financière et par les fantasmes des soldats, mais aussi par le fait que les prostituées « indigènes » sont plus «gentilles » et plus « soumises » que les européennes.

En 1856, dans son tableau de la prostitution en Algérie, le docteur Bertherand explique que l'écrasante majorité des prostituées est constituée d'européennes qui exerçaient précédemment en France et elles sont directement recrutées de Métropole par les tenancières de maisons closes françaises. Pour le docteur Bertherand, ces femmes se prostituent par «oisiveté, goût exagéré pour les toilettes et insuffisance du salaire pour vivre ». Mais les prostituées « indigènes » n’appartiennent pas à cette catégorisation « ces indigènes ». Elles :

sont réunies en Mauresques, Kabyles, Négresses et Juives. Le plus souvent ces femmes sont d'anciennes esclaves et oscillent entre travail domestique mal rémunéré qu'elles accomplissent officiellement. L'illégalisme sexuel officieux qu'elles pratiquent couramment, c'est pour arrondir les fin de mois ${ }^{19}$.

Certaines font partie d'une population traditionnelle mal intégrée à la médina, ce qui place ses femmes nouvellement arrivées, isolées ou déclassées, devant travailler pour vivre dans une situation économique fragile. Le Docteur concède encore : " il faut bien le reconnaitre, la position malheureuse dans laquelle se trouvent un grand nombre de familles indigènes, conduit presque fatalement les femmes à la misère, et au commerce du corps $»^{20}$. Ce sont surtout les activités domestiques qui fournissent en 1910, l'essentiel du travail salarié des femmes. Se-

18 SALARDENNE, Roger. L'Afrique Galante: reportage chez les prostituées juives et mauresques. Paris : Ed Prima, 1930.

${ }^{16}$ Bertherand, A. « De la prostitution à Alger », Mémoires divers. Alger : Typo V. Vailland, 1859, p. 544 .

20 lbid. 
lon G. Laloé, c'est des filles de 12 à 15 ans qui sont employées toute la journée, dans des familles européennes aisées comme domestiques en accomplissant les fonctions domestiques les plus pénibles. Pour Bertherand, la condition de domestique n'est pas loin de la prostitution « la plupart des domestiques ont succombé aux séductions de tout genre qui assaillent les filles privées de la surveillance et des conseils de leurs parents ». A l'époque, selon lui, le un cinquième (1/5) des prostituées d'Alger font profession de domestiques. De plus ces femmes de campagne qui viennent en ville avec leur famille pauvre ou leurs maris qui les répudient sitôt arrivées, se livrent par dépit à la prostitution.

Ce sont généralement les filles soumises « indigènes » qui pallient à la demande sexuelle émanant des militaires qui ne peuvent disposer de maisons closes ou de quartiers réservés. L'armée aménage, alors, sous un contrôle exclusif des structures prostitutionnelles spécifiques : les bordels militaires de campagne (BMC), mis en place dès le début de la conquête algérienne en 1831. L'organisation des BMC demeure assez obscure pendant un siècle, c'est seulement à partir des années 1930 que les archives militaires commencent à fournir des renseignements précis sur leur réglementation. Les BMC maghrébins sont constitués d'équipe de prostituées « indigènes ». En apparence, les prostituées européennes disposaient d'une marge plus importante que les prostituées « indigènes ». Ce sont les hommes « indigènes » qui fréquentaient les filles européennes. Car celles-ci ont toujours exercé une fascination réelle pour eux, et peut-être aussi pour un profond désir de revanche : posséder une femme blanche en la payant comme une vulgaire marchandise permet de renverser le rapport de domination. "Coucher avec une femme blanche était une revanche sur l'histoire coloniale subie, c'était le cas pour moi, mais aussi pour de nombreux algériens que je côtoyais » (disait un ancien combattant).

Lucien Favre, en 1930, décrit les samedi soir et les foules haletantes aux abords des bordels «les arabes prennent la file devant les maisons des prostituées blondes ». Djaffar Lesbert affirmait « permettent de voir l'ailleurs en faisant du surplace ». Mohamed Choukri, illustre cela à travers son roman, Le pain nu, lorsqu'il raconte sa première rencontre au bordel espagnol dans les années 1950, avec une prostituée européenne de Tétouan «qui paraissait bonne et jolie ». La description qu'il en fait est empruntée de surprise d'un jeune homme face à l'altérité sexuelle qui bascule vers le plaisir partagé.

Les chiffres établis en 1951 et 1952 par le docteur Féry dans le département de Constantine, lors du contrôle sanitaire de la prostitution. Sur les 1029 filles soumises répertoriées figurent 970 indigènes et 59 européennes. 856 sont des filles répertoriées et 173 des filles isolées.

\begin{tabular}{|l|l|l|}
\hline Dates & Lieux & $\begin{array}{l}\text { Nombre de prostituées } \\
\text { indigènes }\end{array}$ \\
\hline 1951 & Constantine & 248 \\
\hline 1951 & Sétif & 134 \\
\hline
\end{tabular}




\begin{tabular}{|l|l|l|}
\hline 1951 & Bône & 131 \\
\hline 1951 & Biskra & 91 \\
\hline 1951 & Philipe ville (Skikda) & 70 \\
\hline 1951 & Batna & 79 \\
\hline 1951 & Saint Arnaud (El Eulma) & 67 \\
\hline 1951 & Bordj Bouarreridj & 62 \\
\hline 1951 & Khenchla & 32 \\
\hline 1951 & Guelma & 21 \\
\hline 1951 & Ain El Beida & 17 \\
\hline 1951 & Bougie & 14 \\
\hline 1951 & M'Sila & 13 \\
\hline 1951 & Tébessa & 12 \\
\hline 1951 & Souk Ahras & 11 \\
\hline 1951 & Barika & 10 \\
\hline 1952 & Sidi Belabbès & 200 \\
\hline 1955 & Blida & 200 \\
\hline & & \\
\hline
\end{tabular}

En 1959, d'après le médecin commandant Lazergue, le chiffre est tombé à $88 \%$, alors qu'en même temps la prostitution réglementée a presque doublée (de 79 filles soumises 70 sont de Ouled Nails). Selon Cristelle Taraud (2003), l'origine de cette prostitution est la ville de Messad, l'une des communes de Djelfa. Cette ville apparaît comme le cour de la prostitution soumise, elle irrigue l'ensemble de la région. Les prostituées Ouled Nails sont en situation de quasi monopole à Biskra, Ouled Djellal, Touggourt, Boussaâda, Ouargla et majoritairement à Laghouat et Ghardaïa.

Notre fiction évoque des moments cruciaux de la vie des algériens colonisés et les rôles des femmes. Quelle place les femmes occupent-elles au milieu de ses secousses révolutionnaires qui bouleversent l'univers algérien ? Comment la femme algérienne perçoit-elle la révolution ? La femme algérienne et son corps deviennent un enjeu politique et révolutionnaire important, un champ de bataille prospère aux dirigeants (du FLN) de la guerre de libération. La violence et la guerre s'enracinent dans une sexualité mal vécue surtout du côté algérien car elle relève de l'honneur des hommes :

...Tout le monde a peur, ils marchent tous avec la trouille au ventre. Ces Arabes tellement fiers de leur virilité, de leur courage, qu'ils n'ont jamais cessé de porter l'une et l'autre en bandoulière!... Ils se terrent chez eux comme des rats...mon père me battait, mes frères me battaient, mes oncles et ma mère...Les hommes, les femmes m'ont battu...Et puis un jour, je les ai battus à mon tour. Sur leur propre terrain : celui de l'honneur cher aux Arabes! A présent ils tremblent à l'évocation de mon nom... (CP, p. 60.) 
Criait Dalila, prostituée algérienne au service du colonisateur, à Tahar et Ali vouant une grande aine à sa race et bafouant l'honneur ses siens. Considérés comme des constructions de l'occident, les maisons closes et les quartiers réservés «indigènes» sont vécus par les algériens comme lieux d'oppression et d'humiliation où, à travers les filles soumises, c'est l'ensemble de la communauté nationale qui est dégradée.

C'est la suppression des maisons closes en Métropole en 1946, par la loi (dite Marthes Richard), qui nourrit le sentiment d'une agression contre la famille nationale, car cette loi a été jugée totalement inapplicable en Afrique du Nord, ce qui donne une connotation raciale au problème. Pourquoi l'Algérie, et tout le Maghreb, conservent-ils ces maisons closes, alors que la France les supprime ? Le FLN a commencé des actions de sabotage des maisons closes, des bombes ont explosé au seuil d'une maison de tolérance occupée par des prostituées «algériennes » car ces filles frayent avec les militaires et les civils européens (disaient des informations de l'époque).

En y adjoignant l'idée d'une collaboration active des prostituées au maintien de l'ordre coloniale, la réglementation policière de son côté fait souvent de ces prostituées des indicatrices de la police. Ces filles sont utilisées dans le cadre de la lutte contre les nationalistes. Fragilisées par leur condition, les prostituées résistent difficilement aux actions d'intimidations parfois musclées exercées contre elles. Des fois, c'est par désir de vengeance contre l'ordre traditionnel vécu auparavant dans leur maison parentale que ces filles se rallient à la colonisation. Mais certaines filles soumises « algériennes » se trouvent, soudain, durant cette période, écartelées entre la collaboration avec la France, ou la collaboration avec les nationalistes.

Nous citerons le cas de Dalila, prostituée au service du colonisateur, qui était la cause du massacre de centaines d'hommes « des centaines d'hommes mutilés et tués par sa faute » (CP, p. 37). Dalila, est une danseuse algérienne " aux cheveux teints au henné, qui avaient des reflets pourpres, ils lui tombaient en cascade bouclée sur son dos nu. Son visage malgré le fard criard, conservait un air d'adolescence qui accentuait sa bouche enfantine et rieuse » (CP, p. 56). C'est « une fille assez mystérieuse ; nul ne savait exactement d'où elle venait. Son vrai nom était parait-il Fatma. Un tatouage en forme d'étoile sur son menton dénotait son origine campagnarde » (CP, p. 56). En plus d'être danseuse, elle est la mascotte du deuxième bureau, c'est aussi une prostituée et informatrice du colonisateur, elle se déplace beaucoup, apparait tantôt dans les cabarets d'une grande ville, tantôt dans les fêtes de mess, elle se fait souvent accompagnée dans les jeeps militaires après une soirée passée dans quelques bourgs de la ville.

Dalila représentait un danger. Surtout à l'époque où la purification des mœurs était l'un des principaux jalons de la lutte de libération. L'organisation prohibait aux musulmans de boire de l'alcool, de fumer et de se prostituer. " Ce moyen était infaillible pour une accélération de la prise de conscience politique chez les inconscients... » (CP, p.58.). Le FLN avait mis sa tête à mort à cause de sa trahison 
« depuis des mois, l'organisation cherchait à avoir cette précieuse mascotte du $2^{\text {è }}$ bureau... » (CP, p. 57.). Aussi, « elle se déplaçait beaucoup, apparaissant tantôt dans le cabaret d'une grande ville, tantôt dans les fêtes de mess...Elle se faisait accompagner dans les jeeps militaires après une soirée passée dans quelques bouges de la ville » (CP, p.57.). Elle fut tuée près du cimetière de Bou-H car Le corps de Dalila la prostituée avait projeté la mort des moudjahidines, lui disait Tahar, l'égorgeur du FLN, avec colère : « je t'ai emmenée ici pour que ceux qui furent tués par ta faute et qui reposent en ce lieu soient vengés et entendent tes hurlements de terreur...Ils sont enterrés là, ceux que tu as vendus. » (CP, p. 71). Ce corps exhibé à la prostitution est aussi la cause de sa mort et de sa destruction :

Ce cou si jeune, si lisse...tranché ?... ces cheveux, ce visage, cette jeunesse...c'est une traitresse, elle a causé beaucoup de mal...Ali la maintint contre lui, entourant son corps de son bras. Elle se débattait...Dalila offrait à présent l'image même de l'horreur et de la haine. La sueur collait ses cheveux sur son visage et son cou. Le fard de ses yeux dilué par ses larmes, formait des traces noirâtres sur les joues. Son rouge à lèvres coulait avec la bave...sa robe noire remontée sur ses cuisses ...sa voix avait changé, elle ressemblait à celle d'une vieille femme rongée par la maladie. Une voix rauque, cassée, brisée par les houles des sanglots. (CP, pp. 70-71)

Dans la description, un organe de jouissance peut se transformer en instrument de punition ou en objet d'agression. Le cou, offert aux baisers, élément du corps qui exprime la volupté, est aussi voué à l'égorgement ou à la décapitation. « Ce cou si jeune, si lisse...tranché ? Non, pas ça, mon Dieu ! C'est une traitresse, soit, elle a causé beaucoup de mal...mais elle ne peut pas mourir ainsi. » (CP, p. 70.). " C'est la règle, les musulmans qui trahissent leurs frères, sont égorgés ! Femmes ou hommes. » (Ibid.). Le désir amoureux ne se sépare pas toujours de la pulsion meurtrière.

Les femmes rousses, sont comme des fauves et maléfiques comme Dalila. La chevelure est liée au péché,

Dalila était une fille assez mystérieuse...elle se déplaçait beaucoup, apparaissait tantôt dans le cabaret d'une grande ville, tantôt dans les fêtes de mess. Elle avait récemment fait son apparition dans Baladia agressive et arrogante...ses voisins la craignaient, l'évitaient, allant jusqu'à interdire aux gamins de lui faire des réflexions quand elle se faisait accompagner dans les jeeps militaires...on pouvait croire qu'elle était un danger, surtout à une époque où la purification des mœurs était l'un 
des principaux jalons pour la victoire de la lutte de libération...l'organisation prohibait aux musulmans de boire de l'alcool et de fumer... (CP, pp. 57-58.)

Dalila a les cheveux teints au henné (roux), ils ont des reflets pourpre (couleur du sang) :

Tu as trahi les tiens peut-être pour un collier de pacotille! $\mathrm{Tu}$ es heureuse, insouciante, sans remords...C'est loin l'hiver dernier, pour toi, tout un quartier détruit à cause de ta traîtrise, de ta cupidité...depuis des mois l'organisation cherchait à avoir cette précieuse mascotte du deuxième bureau...ce soir c'est une occasion unique de venger les centaines d'hommes mutilés et tués par sa faute. (CP, p. 57.)

Comme sa robe (rouge et en or). Les femmes aux cheveux roux « duvet de rousse », telle une bête d'or sont des femmes maléfiques. En effet, les cheveux révèlent l'essence profonde des êtres dont le charisme sensuel touche à l'impureté. "L'insolente rougeur, l'onde de feu » caractérisent la chevelure de Dalila, cette femme traitresse, cette femme de l'enfer, cette diablesse, qu'est « la prostituée », « un groupe de paras qui la connaissaient bien, se tenaient près de la scène et lui criaient d'une voix excitée : Aïe, Dalila ! Tu es le Diable ! ... » (CP, p. 57). Ce roux des cheveux, ce rouge sanglant de la robe évoquent des couleurs de l'irrésistible faute et, en filigrane de la pécheresse, la chevelure rousse et la robe rouge au voile d'or sont les couleurs des qualités sexuelles. Sous ses habits, sa chevelure dénouée, rousse, parure maudite se cache la courtisane.

Tahar tenait d'une main un grand couteau de boucherie et de l'autre son pistolet et dit au commissaire - Adjoint Cantini : "nous n'égorgeons pas les soldats ou les policiers par ce que vous faites ce que vous considérez être votre devoir...Si vous nous prenez, vous nous tuez, si nous vous attrapons, nous vous tuons. C'est la règle...Mais les musulmans qui trahissent leurs frères sont égorgés ! Femmes ou hommes ». (CP, p. 70). Dalila face à la mort criait sa haine : « Sale race! Criait-elle, je serai vengée, vous crèverez! Vous ne gagnerez pas la guerre, bande de pouilleux ! Ils sont plus forts que Vous ! ». (CP, p. 71).

Le contraire est vrai, on occulte au-delà du financement du FLN, surtout en Métropole des filles de la rue aidaient les militantes algériennes à faire la collecte pour les cotisations au profit des nationalistes et de la guerre de libération. Elles passaient inaperçues car la police avait l'habitude de les voir dans les rues, dans les bars. Elles pouvaient circuler librement et parler à tous de manière normale. Elles étaient aussi pour les nationalistes de véritables sources de renseignements, car elles avaient des contacts avec des policiers et des militaires français. Yacef Saadi signale aussi le rôle joué par certaines d'entre elles au moment de la bataille d'Alger : 
Les prostituées frissonnaient à la moindre bribe mettant en cause les Fidais, vibraient à la plus petite particule concernant des préparatifs de bouclages, de rafles ou d'opérations de ratissage imminentes, on ne peut imaginer le nombre de fois que des scoops de première importance nous sont parvenus grâce au double sacrifice de ces cloîtrées. ${ }^{21}$

Certaines sont de belles figures de résistance dont les actions ont permis de cacher, parfois de sauver des militants nationalistes blessés ou en cavales. Car le colonisateur s'est attaqué, en cette période, aux femmes algériennes (combattantes ou prostituées) par des moyens de répression, de torture perpétrant contre elles des violences physiques et sexuelles: viols atroces: «Ma fiancée était infirmière...Elle avait vingt et un ans...Un jour qu'elle se trouvait auprès d'un blessé caché chez elle, les soldats firent irruption. Ils saccagèrent tout, massacrèrent les femmes...Elle fut violée et rouée de coup jusqu'à en succomber » $(\mathrm{CP}, \mathrm{pp}$. 4142.), ainsi que d'autres humiliations et fantasmes du voile ôté comme : «le geste stupidement théâtral de Haïk, de femmes mis en lambeaux par celles qui le portaient et dont on fait, tout le monde sait qu'il s'agit de pensionnaires de maisons closes...les pieds noirs exultaient » (CP, p.187).

A ce propos, Fanon montre comment le voilement, puis le dévoilement ont été utilisés par les femmes algériennes pour déjouer la surveillance française et participer activement à la révolution. Elles sont actrices de la révolution à part entière. Pour les français une femme dévoilée est nécessairement acquise à la cause française et la femme voilée est totalement inoffensive et incapable de participer à la lutte armée. Ce qui est complètement faux car les poseuses de bombes étaient dans un début voilées puis, elles se sont dévoilées une fois leurs actions découvertes.

Ces actions de prostituées patriotes dont il est difficile de mesurer l'ampleur, ne remettent pas en cause l'habitus entre nationalisme et prostitution qui tourne essentiellement autour de la question de «la collaboration corporelle et charnelle » des filles algériennes prostituées d'une part, avec les européens, jouant le rôle de passerelles, brouillant les frontières du pouvoir colonial. Et d'autre part, avec les nationalistes algériens jouant le rôle de libératrices des pulsions accumulées par les Moudjahiddines ou Fidais. Comme c'est le cas du personnage de Houria dans Ciel de Porphyre. Tahar et Ali

sont arrivés devant une maison non loin de sa baraque (Tahar). Une vieille femme nous a ouvert la porte. Son visage s'est illuminé en reconnaissant Tahar. Il l'a prise tendrement

${ }^{21}$ YACEF, Saad. La bataille d'Alger. Alger : Editions Casbah/Publisud, 1997. p. 154. 
par le cou, lui murmurant quelques mots auxquels la femme a répondu par un hochement affirmatif de tête. (CP, p. 223.).

Ali disait :

Une jeune femme entre, elle porte un pantalon bleu, assez grande, avec quelque chose de sensuel dans sa démarche traînante. Son visage est nimbé d'un charme profond...Son visage est parfait. Elle est belle... Tout dans son attitude réservée et naturelle à la fois me paralyse. Là bas chez Nini, c'était plus facile ! (CP, p. 224).

Son nom surpris Ali : «...Quel étrange nom! Houria...Hourie...Liberté...Ange...Oui en vérité, en ces temps de haine, de peur, et de mort, une femme comme elle était tout un symbole... » (CP, p. 224.). C'est une jeune femme belle, mince, Ali la compare aux prostituées européennes de chez Nini : « elle n'a rien de commun avec les femmes de là bas, qui pour la plupart sont grasses et vulgaires. Chez Houria, tout est délié, racé et fin... un mot...un poème, une rose, une démarche...un geste...un regard...un air de musique » $(\mathrm{CP}, \mathrm{p}$. 224.). Ali se sent fondre devant elle dans une douce émotion. Et pourtant, Houria est la prostituée des combattants, elle préfère aimer et consoler, c'est cela son travail auprès de l'organisation. Il est « curieux de la réaction de cette femme, pourquoi porte-t-elle cet air de tristesse sur son visage ? ». Il se demande aussi pourquoi Houria pratique ce métier. " Pourquoi ne travailles - tu pas ? 》 demande Ali. « Je préfère aimer et consoler, c'est cela mon travail » répondit Houria. « Jusqu’à quand continueras -tu ces actes de consolations amoureuses comme tu dis?» demande Ali. Elle répond « jusqu'au jour où cette terre sera libre. Alors pour elle et pour moi finira le temps de la honte... ».

Houria lie son destin à celui de son pays, l'Algérie : «Et si un jour mon pays était encore floué une autre fois, alors je reprendrai le chemin de « consolations amoureuses », c'est ainsi que je porterai le deuil de la dignité ». (CP, p. 228.). Houria par sa contribution généreuse procure à Ali : abandon, tendresse, repos et partage «je suis en train de découvrir la tolérance et la compréhension de l'autre ». (CP, p. 226.).

La prostituée Dalila qui vit dans une triple situation paratopique qui passe d'une situation minimale, car dans son village, son père la battait, ses frères, ses oncles et même sa mère la battaient « les hommes, les femmes m'ont battue... » (CP, p. 6o.), à une situation maximale : «... Et un jour, je les ai battus à mon tour sur leur propre terrain : celui de l'honneur...cher aux Arabes ! A présent, ils tremblent à l'évocation de mon nom...Et moi je n'ai jamais eu peur, je n'ai pas peur...de personne ! » (Ibid.), elle est devenue belle, élégante " c'était un spectacle fascinant que la souplesse extraordinaire de cette fille »; et puis de sa grande beauté qui laissait croire qu'elle est 
un grand danger, elle bascule de cette situation maximale acquise au contact des militaires français à une situation minimale d'indicatrice pour le colonisateur et de traitresse pour ses compatriotes: «tu as trahi les tiens peut-être pour un collier de pacotille ! Tu es heureuse, insouciante, sans aucun remords...tout un quartier détruit à cause de ta traitrise, de ta cupidité... » (CP, p. 57.)

Par contre, Houria passe d'une situation minimale de prostituée, de femme portant le deuil de la dignité, une femme de « consolation amoureuse » à une double situation maximale. Dans un premier temps, cette femme symbole, Houria, Hourie, liberté et Ange...jouant à la consolatrice des membres du FLN durant la colonisation : « je préfère aimer et consoler...c'est cela mon travail... Et jusqu'au jour où cette terre sera libre...alors pour moi finira le temps de la honte...je me marierai alors peut-être. ». Houria sera une femme libre, après l'indépendance, elle devient une femme élégante dans un tailleur blanc, avec de longs colliers dorés, dans un second temps, «tout dans son apparence dénote l'aisance matérielle » (CP, p. 309.), elle monte dans sa voiture, une 504 blanche, elle s'est marié à l'indépendance...

Nous concluons dans un premier temps, que la question nationale, durant la guerre de libération, était liée à des « enjeux de sexe ${ }^{22}$ disait E. Dorlin. Les prostituées étaient sommées de choisir leurs camps. Christelle Taraud résume bien cela « par le métissage sexuelle, social, religieux et linguistique dans lesquelles elles se sont engagées, ces prostituées « indigènes ", ont dès lors constitué un groupe médian, faisant lieu entre les différents « fronts communautaires » $»^{23}$.

Dans un second, on a démontré que le corps et le décor entretenaient des rapports intimes et témoignaient de la cohérence d'un imaginaire et d'un inconscient. La description des prostituées dans la maison close «La sourie noire », tenue par madame Nini, dans Ciel de Porphyre, démontait cette relation :

Un grand salon avec un fond, un bar impressionnant. Les murs sont décorés de tableaux représentant des femmes dévêtues de manière suggestive. Les filles à moitié nues dans des poses aguichantes, sont dispersées dans des divans ajoutant une note sensuelle à l'atmosphère. Tout contribue à créer une ambiance très érotique. (C P, p. 138.).

Cette déchéance sociale, durant la période coloniale, avait crée un environnement de malaise où régnaient tous les problèmes qui entravaient l'épanouissement sain des peuples. Lemsine conceptualise le phénomène de la prostitution en relation avec les figures de Dalila/Houria et du traitement du corps par rapport à la sexualité et au pouvoir.

22 DORLIN, Elsa. La matrice de la race. Généalogie sexuelle et coloniale de la Nation française. Paris : La Découverte, 2009.

${ }^{23}$ TARAUD, Christelle. "Amour interdit" Marginalité, prostitution, colonialisme (Maghreb, 1830 1962). Paris : Payot, 2012. 
Notre intervention a essayé de démontrer comment le corps des femmes algériennes est entré en guerre, en révolution contre une colonisation sexuelle et raciale. Les prostituées du FLN n'ont jamais été considérées comme objets ou marchandises à consommer mais comme forces disantes, pesantes, agissantes. De véritables actrices auprès desquelles « $\mathrm{Ali}$ » découvre son humanité car elles étaient tolérantes et compréhensives faisant don de leurs corps, telle Houria, l'ange gardien des nationalistes algériens.

\section{RÉFÉRENCES BIBLIOGRAPHIQUES}

ALOULA, Malek. Le Harem colonial. Images d'un sous érotisme. Paris : Ed Séguier, 1981.

AZIZ, Germaine. Les Chambres closes. Paris : Ed Stock, 1980.

BEAUGE, Florence «Le tabou du viol des femmes pendant la guerre d'Algérie Commence à être levé ». Le Monde, 11 octobre 2001.

BERTHERAND. Adolphe. "De la prostitution à Alger ». In : Mémoires divers. Alger : Typo V. Vailland., 1859.

CORBIN, Alain. Les Filles de noces. Misère sexuelle et prostitution au $19^{\text {ès }}$ et au $20^{\grave{e}}$ s. Paris : Flammarion, 1978.

DIDIER, ERIBON. Le Corps politique. Quelques réflexions à propos du $40^{\text {ème }}$ anniversaire des émeutes de Stonewall.

DORLIN. Elsa. La Matrice de la race. Généalogie sexuelle et coloniale de la Nation française. Paris : La Découverte, 2009.

FANON. Frantz. L’An V de la révolution algérienne. Paris : Ed Maspero, 1960.

FROMENTIN, Eugène. Une année dans le Sahel. Paris : Ed Plon, 1848.

LEMSINE, Aïcha. Ciel de Porphyre. Paris : J.-C. Simoën, 1978.

MOLINIER, Pascale, Autre chose qu'un désir de peau... Le Nègre, la Blanche et le Blanc dans deux romans de Dany Laferrière in (Dorlin éd) Sexe, Race, classe. Pour une épistémologie de la domination. Paris : Puf, 2009. 
MONTAGU, Lady Mary. L'Islam au péril des femmes nues. Une Anglaise en Turquie au $18^{\text {è }}$ S. 2001.

NORBERT, Elias. La Société de cours. Paris : Champs essais, 1985.

SALARDENNE, Roger. L'Afrique Galante : reportage chez les prostituées juives et mauresques. Paris : Ed Prima, 1930.

TARAUD, Christelle. "Amour interdit" Marginalité, prostitution, colonialisme (Maghreb, 1830-1962). Paris : Payot, 2012.

La Prostitution coloniale. Algérie, Tunisie, Maroc (18301962). Paris : Payot, 2003.

YACEF, Saâdi. La Bataille d’Alger. Alger : Éditions Casbah/Publisud, 1997. 